\title{
IMPACT OF COMORBIDITIES IN MEXICAN SARS-COV-2-POSITIVE PATIENTS: A RETROSPECTIVE ANALYSIS IN A NATIONAL COHORT
}

\begin{abstract}
Ashuin Kammar-García ${ }^{1,2}$, José de J. Vidal-Mayo ${ }^{1}$, Juan M. Vera-Zertuche ${ }^{3}$, Martín LazcanoHernández ${ }^{4}$, Obdulia Vera-lópez ${ }^{4}$, Orietta Segura-Badilla ${ }^{5}$, Patricia Aguilar-Alonso ${ }^{4}$, and Addi R. NAVARRO-CRUZ ${ }^{4 *}$

${ }^{1}$ Emergency Department, Instituto Nacional de Ciencias Médicas y Nutrición Salvador Zubirán (INCMNSZ), Mexico City, Mexico; ${ }^{2}$ Sección de Estudios de Posgrado e Investigación, Escuela Superior de Medicina, Instituto Politécnico Nacional, Mexico City, Mexico; ${ }^{3}$ Department of Endocrinology, Obesity Clinic, INCMNSZ, Mexico City; ${ }^{4}$ Deparment of Biochemistry and Foods, Faculty of Chemical Sciences, Benemérita Universidad Autónoma de Puebla, Pue., Mexico; ${ }^{5}$ Department of Nutrition and Public Health, Faculty of Health and Food Sciences, "Programa Universidad Bío-Bío (UBB) Saludable," Universidad del Bío-Bío, Concepción, Chile
\end{abstract}

\begin{abstract}
Background: The coronavirus disease 2019 outbreak is a significant challenge for health-care systems around the world. Objective: The objective of the study was to assess the impact of comorbidities on the case fatality rate (CFR) and the development of adverse events in patients positive for severe acute respiratory syndrome coronavirus 2 (SARS-CoV-2) in the Mexican population. Materials and methods: We analyzed the data from 13,842 laboratory-confirmed SARS-CoV-2 patients in Mexico between January 1, 2020, and April 25, 2020. We investigated the risk of death and the development of adverse events (hospitalization, pneumonia, orotracheal intubation, and intensive care unit [ICU] admission), comparing the number of comorbidities of each patient. Results: The patient mean age was $46.6 \pm 15.6$ years, $42.3 \%(n=5853)$ of the cases were women, $38.8 \%$ of patients were hospitalized, $4.4 \%$ were intubated, $29.6 \%$ developed pneumonia, and $4.4 \%$ had critical illness. The CFR was $9.4 \%$. The risk of hospitalization (odds ratio $[\mathrm{OR}]=3.1,95 \%$ confidence interval $[\mathrm{Cl}]: 2.7-3.7$ ), pneumonia (OR $=3.02,95 \%$ $\mathrm{Cl}: 2.6-3.5$ ), ICU admission ( $\mathrm{OR}=2,95 \% \mathrm{Cl}: 1.5-2.7$ ), and CFR (hazard ratio $=3.5,95 \% \mathrm{Cl}: 2.9-4.2$ ) was higher in patients with three or more comorbidities than in patients with 1,2 , or with no comorbidities. Conclusions: The number of comorbidities may be a determining factor in the clinical course and its outcomes in SARS-CoV-2-positive patients. (REV INVEST CLIN. 2020;72(3):151-8)
\end{abstract}

Key words: Coronavirus disease 2019. Comorbidities. Mortality. Adverse events. Severe acute respiratory syndrome coronavirus 2. Demographic characteristic.

*Corresponding author:

Addi Rhode Navarro-Cruz

E-mail: addi.navarro@correo.buap.mx
Received for publication: 07-05-2020

Approved for publication: 25-05-2020

DOI: $10.24875 / R I C .20000207$

0034-8376 / (C) 2020 Revista de Investigación Clínica. Published by Permanyer. This is an open access article under the CC BY-NC-ND license (http://creativecommons.org/licenses/by-nc-nd/4.0/). 


\section{INTRODUCTION}

In early December 2019, a group of cases of "pneumonia of unknown origin" was reported in Wuhan, capital of the Chinese province of Hubei ${ }^{1}$. Over the next 2 months, the outbreak spreads rapidly throughout China, and the severe acute respiratory syndrome coronavirus 2 (SARS-CoV-2) was identified shortly after as the responsible pathogen ${ }^{2}$. What was initially an epidemic quickly spread to the rest of the world, declared as a global pandemic and named coronavirus disease 2019 (COVID-19) 2 .

The presence of any comorbidity had previously been shown to condition an increased risk of developing acute respiratory distress syndrome in patients with influenza ${ }^{4}$. In the first reports of SARS-CoV-2 disease, $32 \%$ of confirmed patients had concomitant comorbidities and among them, patients admitted to the intensive care unit (ICU) had significantly more ${ }^{5,6}$. Many elderly patients who became seriously ill had evidence of underlying disease, of cardiovascular, hepatic, and/or kidney origin or malignant tumors, and they often died as a result of their original comorbidities ${ }^{5}$. Other studies have reported that overweight and obese patients are at increased risk of admission to the ICU and of a fatal outcome ${ }^{2}$.

COVID-19 is an ongoing global pandemic, without a vaccine or effective treatment in the near horizon, so only public health measures may potentially decrease its impact; therefore, all the original comorbidities of individuals infected with SARS-CoV-2 must be accurately assessed ${ }^{7}$.

Since comorbidities could be a risk factor for adverse outcomes, the objective of this study is to assess their impact on the case fatality rate (CFR) and on the development of adverse events in patients positive for SARS-CoV-2 in the Mexican population.

\section{MATERIALS AND METHODS}

This retrospective, observational study was conducted with a multicenter national cohort of 13,842 patients positive for SARS-CoV-2 in Mexico, between January 1, 2020, and April 25, 2020. The data were obtained from the General Directorate of Epidemiology of the Mexican Ministry of Health that updates daily an open-source data set with information on patients with a suspicious, negative, and definitive diagnosis of COVID-198.

This study included patients with a confirmed diagnosis of COVID-19 based on a positive result of the SARS-CoV-2 test by real-time reverse transcription polymerase chain reaction, certified by the National Institute of Epidemiological Diagnosis and Reference. Data were obtained from different medical units in the 32 Mexican states that belong to 14 different institutions integrating the Mexican health sector. The collection of patient data from each medical center caring for patients with COVID-19 is forwarded to the Ministry of Health of Mexico and, once validated, it is uploaded to the epidemiological surveillance platform, and real-time patient data are updated in the cohort daily.

All demographic data (age, origin, sex, nationality, pregnancy, smoking status, date of symptom onset, date of medical attention, contact with another confirmed case, and comorbidities) and clinical data (onset of symptoms, presence of pneumonia, requirement for orotracheal intubation, and the need for intensive care) were collected on arrival at the medical center for hospital care. Depending on the clinical criteria, patients were admitted to a hospital area to continue their treatment and observation or were discharged with outpatient treatment. The death date was updated daily.

Comorbidities were determined by self-report at the time of medical care and classified as present or absent. The defined comorbidity groups were diabetes, chronic obstructive pulmonary disease, asthma, immunosuppression, hypertension, cardiovascular disease (CVD), obesity, and chronic kidney disease (CKD). Comorbidities were classified according to their number in every individual into the following categories: without comorbidities, 1 comorbidity, 2 comorbidities, and $\geq 3$ comorbidities.

The primary endpoint was all-cause of death during follow-up, and secondary endpoints were the presence of adverse events defined as hospitalization, the development of pneumonia, intubation, and ICU admission. 


\section{Statistical analysis}

Data are presented as frequencies and percentages in the case of categorical variables and as means \pm standard deviation for continuous or discrete variables. Comparisons were made with the Chi-square test, Student's t-test, and one-way ANOVA with Tukey test post hoc analysis. The impact of the number of comorbidities on overall survival was analyzed with the Kaplan-Meier methods with pairwise comparisons and between categories with the log-rank test. Multivariate logistic regression analysis was applied to determine the risk of adverse events for pre-existing comorbidities. Multivariate Cox proportional hazards regression models determined the prediction of the CFR in patients with COVID-19. Variables were entered into the multivariate models at an initial significance level of $p<0.1$ in the bivariate analysis, using the Enter method to establish the independent contribution of each covariate on adverse events or CFR. Multivariate models were adjusted by sex, age, smoking status, and time from onset of symptoms to initial care. The multivariate-adjusted Cox regression models were rerun in three subgroup analyses: in hospitalized patients, intubated patients, and patients admitted to the ICU. $p<0.05$ was considered statistically significant. All analyses were performed in the SPSS statistical program version 21 and GraphPad Prism version 6 .

\section{RESULTS}

Most of the positive cases for SAR-CoV-2 were men $(57.7 \%)$ with a mean age of $46.6 \pm 15.6$ years, and $98.7 \%$ of cases were Mexican. The prevalence of COVID-19 increases with age. More than a quarter of current cases were in contact with a confirmed case of COVID-19 (Table S1).

Of 13,842 confirmed cases, 5373 (38.8\%) required hospitalization, and $67.7 \%(n=3635)$ of these patients had pneumonia on admission. Among the hospitalized patients, $11.4 \%(n=611)$ required orotracheal intubation and $55.5 \%(n=339)$ of these were admitted to ICU. The total CFR was $9.4 \%$ (men: $11.1 \%$, women: $7.1 \%, p<0.0001$ ), but the CFR in COVID-19 hospitalized patients was $21.9 \%$, and the rates in patients who required orotracheal intubation and in those who required intensive care were $50.2 \%$ and $41.5 \%$, respectively.

Among the SARS-CoV-2-positive cases, $45.3 \%$ had at least one comorbidity. About $26 \%$ of the patients had 1 comorbidity, $12.9 \%$ had 2 comorbidities, and $6.4 \%$ had $\geq 3$ comorbidities. The patients' age increased according to the number of comorbidities (without comorbidities: $41.6 \pm 14.3,95 \%$ confidence interval (Cl): 41.3-41.9; 1 comorbidity: $49.6 \pm 14.9,95 \% \mathrm{Cl}$ : 49.1-50.1; 2 comorbidities $52.3 \pm 14.1,95 \mathrm{Cl} \%$ : 54.6-55.9; and $\geq 3$ comorbidities: $59.1 \pm 13.5$, $95 \%$ Cl: $58.2-60.01 ; p<0.0001)$. The proportion of patients who developed adverse events increased with the number of comorbidities and was higher in the groups with two and three or more comorbidities (Table S2).

Survival analysis showed that $95.6 \%$ of patients without comorbidities survived, while in patients with 1 comorbidity ( $88.5 \%), 2$ comorbidities ( $81.8 \%$ ), and $\geq 3$ comorbidities ( $73.7 \%$ ), survival was statistically decreased (log-rank Mantel-Cox, $p<0.0001$ ). The greater the patients' age, the lower the survival ( $\leq 20$ years: $98.8 \% ; 21-30$ years: $99 \%, 31-40$ years: $97.1 \%$; $41-50$ years: $92.6 \%$; $51-60$ years: $88 \%$, and $>60$ years: $76.3 \%$; log-rank Mantel-Cox, $p<0.0001$ ). When survival analysis was performed according to the number of comorbidities and per age group, we observed that comorbidity determines survival regardless of age since it decreases even in younger cases (Fig. 1). Similarly, in hospitalized patients, in those who required orotracheal intubation, and in those who required intensive care, survival was inversely proportional to the number of comorbidities: hospitalized patients: without comorbidities: $85.5 \%$, 1 comorbidity: $77.7 \%$; 2 comorbidities: $71.2 \%$; and $\geq 3$ comorbidities: 65.5\%; (log-rank Mantel-Cox, $\mathrm{p}<0.0001$ ); intubated patients: without comorbidities: $58.1 \%$; 1 comorbidity: $49.5 \%$; 2 comorbidities: $40.3 \%$; and $\geq 3$ comorbidities: $46.7 \%$; (log-rank Mantel-Cox, $\mathrm{p}=0.002$ ) and; ICU patients: without comorbidities: $66.4 \%$; 1 comorbidity: $59.4 \%$; 2 comorbidities: $49.1 \%$; and $\geq 3$ comorbidities: $48.1 \%$; (log-rank Mantel-Cox, $\mathrm{p}<0.0001$ ).

Regression analysis established that the presence of comorbidities increases the risk of hospitalization, development of pneumonia, the requirement for orotracheal intubation, ICU admission, and the CFR. 
Figure 1. Survival curves according to age ranges and number of comorbidities. A: patients < 20 years, B: patients between 21 and 30 years, C: patients between 31 and 40 years, D: patients between 41 and 50 years, E: patients between 51 and 60 years, F: patients $>60$ years.
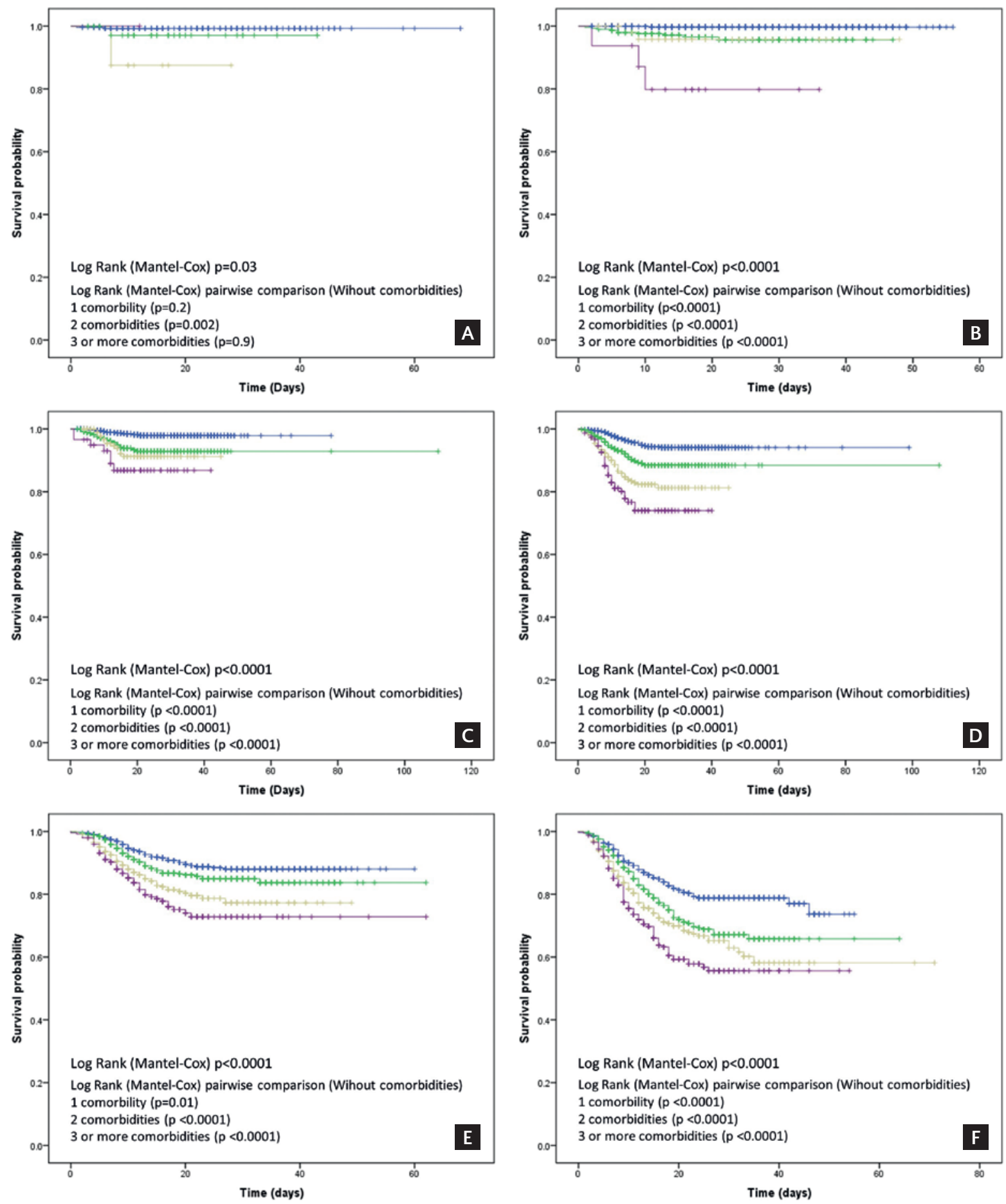

\begin{tabular}{|c|c|c|c|c|c|c|c|c|c|c|c|c|}
\hline \multicolumn{13}{|l|}{ Survival probabilities } \\
\hline \multirow[b]{2}{*}{ Number of comorbidities } & \multicolumn{2}{|c|}{$\leq 20$ years } & \multicolumn{2}{|c|}{$21-30$ years } & \multicolumn{2}{|c|}{$31-40$ years } & \multicolumn{2}{|c|}{ 41-50 years } & \multicolumn{2}{|c|}{$51-60$ years } & \multicolumn{2}{|c|}{$>60$ years } \\
\hline & $\mathrm{n}$ & $\%$ & $\mathrm{n}$ & $\%$ & $\mathrm{n}$ & $\%$ & $\mathrm{n}$ & $\%$ & $\mathbf{n}$ & $\%$ & $\mathrm{n}$ & $\%$ \\
\hline$\neg$ Without comorbidities & 282 & 99.3 & 1456 & 99.7 & 2013 & 98.5 & 1793 & 95.9 & 1080 & 91.4 & 615 & 83.9 \\
\hline$\square 1$ comorbility & 36 & 97.3 & 285 & 96.6 & 679 & 94.7 & 813 & 91.3 & 738 & 88.4 & 638 & 77 \\
\hline-2 comorbidities & 7 & 87.5 & 52 & 96.3 & 190 & 93.6 & 341 & 85.3 & 409 & 83.6 & 456 & 73.1 \\
\hline$\Omega 3$ or more comorbidities & 1 & 100 & 13 & 81.3 & 52 & 88.1 & 119 & 78.8 & 195 & 78.6 & 274 & 66.5 \\
\hline
\end{tabular}


Figure 2. Multivariate analyses of different groups of patients according to the number of comorbidities. Model adjusted for age, sex, and time from onset of symptoms to initial care, the hazard ratio and $95 \%$ confidence interval are compared with those obtained in patients with no associated comorbidity.

Adj. Hazard Ratio (95\%Cl)

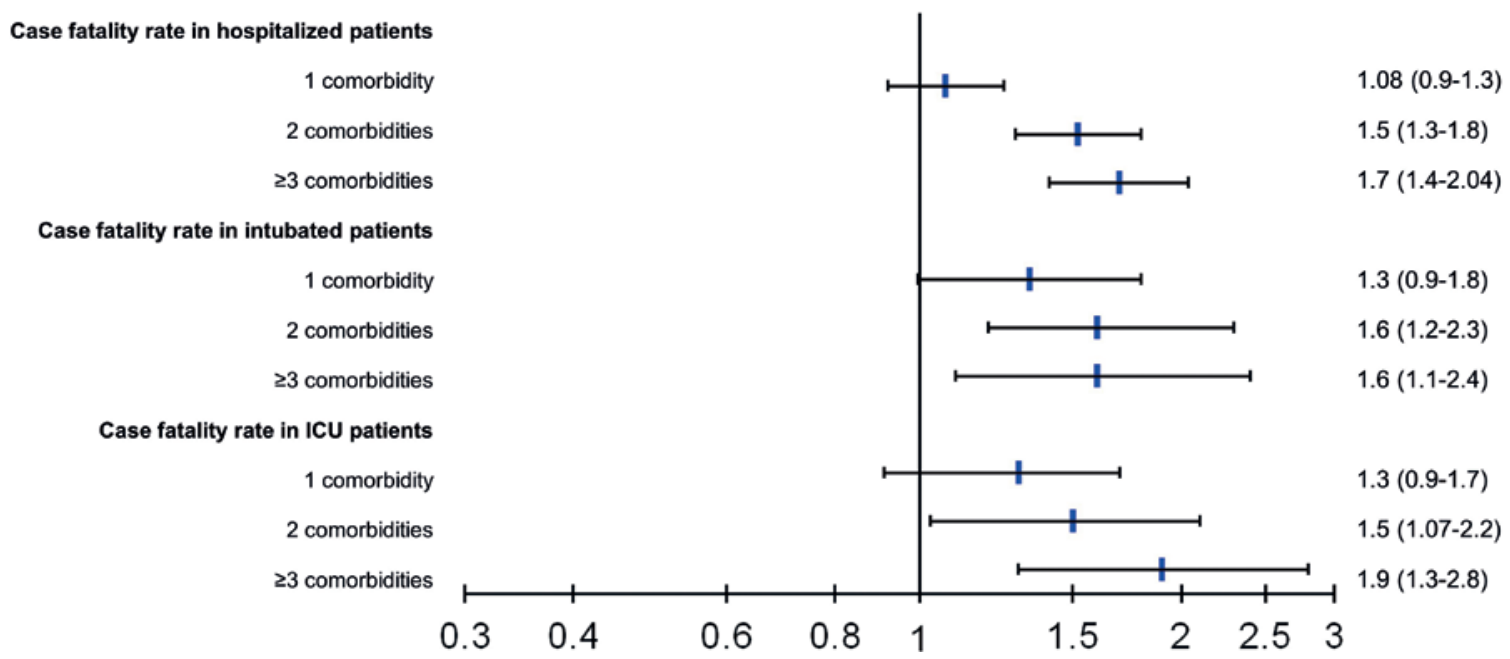

Diabetes, hypertension, and obesity were the comorbidities established as risk factors for all outcomes. Patients with three or more comorbidities have a higher risk of developing adverse events in comparison with cases with two or one comorbidities; further, compared with those without any comorbidity (Table S3), the combination of diabetes and obesity was the most significant in all outcomes, and diabetes and CKD led to an increased risk of hospitalization, pneumonia, and CFR (Table S4). Different subanalyses were performed in patient subgroups to predict the CFR in hospitalized, intubated, and ICU patients; we observed that the number of comorbidities remains a risk factor for the CFR in SARS-CoV-2-positive patients (Fig. 2).

\section{DISCUSSION}

This study analyzes the demographic characteristics of the infected Mexican population and the impact of the number of comorbidities on the development of adverse events and the CFR in SARS-CoV-2-positive patients in Mexico. The most frequent comorbidities in this population were hypertension, obesity, and diabetes, similar findings to those observed in China at the onset of the pandemic 5,9,10,11.

We found that $45.3 \%$ of the cases had at least one comorbidity. Italy reported that the vast majority of patients who died from COVID-19 until April 2, 2020, had chronic comorbidities (2.7 diseases on average): $97.2 \%$ of patients had at least one comorbidity, $51.3 \%$ had three comorbidities, $23.9 \%$ two comorbidities, and $22.1 \%$ one comorbidity. Hypertension was the most common comorbidity in $72.1 \%$ of patients followed by diabetes mellitus in $31.5 \%$ and ischemic heart disease in $27.4 \%$ of cases $^{12,13}$.

The effect of comorbidities on fatality rates is wellknown; however, in Mexico, this takes on a new and alarming dimension since the country ranks second in the world in obesity prevalence ${ }^{14}$; according to the ENSANUT 2018 survey ${ }^{15}, 75.2 \%$ of the Mexican population over the age of 20 is overweight or obese. Furthermore, the prevalence of diabetes in Mexicans above the age of 20 is $10.3 \%$ ( 8.6 million individuals) and the prevalence of hypertension is $18.4 \%$ in patients over 20 years of age and particularly, in those above the age of 70 , with a prevalence of $26.7 \%$. This prevalence of metabolic diseases characterizes Mexico as an extremely vulnerable country to the development of complications caused by COVID-19.

Similar to our findings, multiple studies have been conducted to determine the risk factors associated with the development of critical illness requiring mechanical ventilation and leading to death in patients with COVID-19. Thus, the following are consistently 
identified as the main risk factors for severe disease: age over 65 years, chronic lung disease, systemic arterial hypertension, CVD, diabetes mellitus, obesity (body mass index $[\mathrm{BMI}] \geq 30$ ), immunosuppression, end-stage CKD, and liver disease ${ }^{1,5,10,16-18}$.

Diabetes, hypertension, and obesity were the only comorbidities that were statistically significant in all models analyzing adverse events in this cohort of Mexican patients, suggesting that metabolic diseases are a determining factor in the severity of COVID-19. Of these three, only obesity was unaffected by the presence of other risk factors after multivariate adjustment, which would suggest an association between abdominal adiposity and disease severity.

In the context of SARS-CoV-2 infection, BMI has been reported to be significantly higher in critically ill patients compared with other COVID-19 patients $(27 \pm 2.5 \text { vs. } 22 \pm 1.3 ; p<0.001)^{19}$. Another study established that the BMI of the group of patients with COVID-19 and critical illness was higher than that of patients without critical illness (25.5, interquartile range [IQR]: $23-27.5$ vs. 22.0, IQR: $20-24$, $\mathrm{p}=0.003$ ) and that $88.2 \%$ of the patients who died from COVID-19 had a BMI above $25^{20}$.

A study in France showed that the risk of requiring invasive mechanical ventilation (IMV) in patients with COVID-19 and with a BMI $>35$ is 7 times higher than in patients with a $\mathrm{BMI}<25^{21}$. In New York, patients with a BMI of $30-34$ (odds ratio $[O R]=1.8,95 \%$ $\mathrm{Cl}: 1.2-2.7)$ and patients with a $\mathrm{BMI}>35(\mathrm{OR}=3.6$, $95 \% \mathrm{Cl}: 2.5-5.3)$ had a greater risk of requiring admission to the ICU than patients with a $\mathrm{BMI}<30^{22}$. When survival analyses were performed according to the number of comorbidities in each age group, we observed that comorbidity determines survival regardless of age since it decreases it, even in the youngest cases. In the age group between 21 and 30 years in which survival is $99 \%$, in patients with three or more comorbidities, it decreases almost $20 \%$ and since most of the Mexican population harbors the three most frequent comorbidities in COVID-19 cases (arterial hypertension, obesity, and diabetes mellitus), it appears that there is a high disposition to the development of adverse events in the Mexican population. This fact could perhaps be related to the chronic pro-inflammatory state associated with obesity and the metabolic syndrome, which favors a prothrombotic and pro-inflammatory environment with higher tissue expression of angiotensin-converting enzyme 2, a protein associated to the binding of SARS-CoV-2 in the alveolar epithelium ${ }^{23}$. Furthermore, adipose tissue has proven to be a viral reservoir for other pathogens such as Ad-36 adenovirus, influenza A virus, HIV, cytomegalovirus, Trypanosoma gondii, and Mycobacterium tuberculosis; hence, SARS-CoV-2 could remain viable in the adipose tissue of these patients ${ }^{24}$.

The population of critically ill patients with COVID-19 represented $4.4 \%$ of all cases $(n=611)$ and $11.4 \%$ of these cases were hospitalized, a similar proportion to that reported in other populations with this disease, such as New York ${ }^{25}$, Lombardy, ${ }^{26}$ and China ${ }^{17}$. Moreover, in a study of 1043 patients admitted to the ICU, $68 \%$ had at least one comorbidity, with hypertension being the most frequent $(49 \%)$, followed by CVD ( $21 \%)$, hypercholesterolemia ( $18 \%$ ), and diabetes mellitus (17\%). It should be noted that in this study, age as the only risk factor was not found to be a significant variable in terms of requiring admission to the ICU26.

Results in other cohorts ${ }^{13,20,26}$ have reported that their general CFR is lower than that of our cohort in Mexico (9.4\% vs. $2.4-7.2 \%$ in China and Italy, respectively). However, the CFR in patients who were hospitalized and critically ill was proportionately similar to that reported in other international cohorts, underscoring the fact that in the group of critically ill patients, it is approximately $50 \%$, according to various published series ${ }^{17,27,28}$. There is, however, wide variation in the CFR reported in the subgroup of patients with critical illness and requiring in vitro maturation, ranging from $26 \%$ to $97 \%{ }^{16}$. These variations may be related to the geographical location of hospital centers, their capacity, the availability of ICU, the implementation of specific care protocols in this group of patients, and the specific characteristics of each population. Besides, several of these studies have reported results on patients who were still hospitalized at the time of publication ${ }^{13,25,26}$. Compared with data obtained in the Mexican population during the influenza A H1N1 virus pandemic in $2009^{29}$, the case fatality found in our study in critically ill patients is higher ( $50.2 \%$ in COVID- 19 vs. $41.4 \% \%$ in $\mathrm{H} 1 \mathrm{~N} 1$ influenza), which underscores the impact that this disease has 
had on the Mexican population and the major challenge it represents for the country's health system.

Another result that should be emphasized in this cohort is that only $55.5 \%$ of critically ill patients on IMV were admitted to ICUs; this may be a consequence of the limited availability of beds in critical care units in the national health system (approximately 5200 beds in the country, for a population of just over 126 million inhabitants). This point is relevant, since in a retrospective cohort of patients who died from COVID-19 in China (that has 3.6 intensive care beds per 100,000 inhabitants), the management of this type of patients by a medical team that is not led and coordinated by intensive care physicians and the delay in the implementation of IMV is probably associated with unfavorable outcomes ${ }^{30}$.

The main limitation of this study is the validation of the database since we did not directly collect the data included in the database and therefore could not corroborate each of the analyzed variables; all datasets were directly reviewed and validated only by the Mexican Ministry of Health. Other limitations were failure to report the initiation dates of each adverse event, the fact that self-reporting comorbidities could lead to underreporting of cases, particularly since many are subclinical and lead to underdiagnosis, as well as the lack of information on hospital discharges and the underreporting of COVID-19 cases in the Mexican population.

In conclusion, patients with comorbidities are at greater risk of developing adverse events, and their CFR is also increased when compared with previously healthy patients. The number of comorbidities could be a determining factor in the patients' clinical course and outcomes in cases that are positive for SARS-CoV- 2 . These findings allow us to identify areas of opportunity on which to focus research, improve the quality of information, as well as the clinical outcomes in Mexican patients with COVID-19.

\section{ACKNOWLEDGMENTS}

The authors would like to thank MML. Estherly María Solis-Rodríguez from the Instituto Politécnico Nacional, Mexico for the English grammar and style correction.

\section{SUPPLEMENTARY DATA}

Supplementary data are available at Revista de Investigación Clínica online (www.clinicalandtranslationalinvestigation.com). These data are provided by the corresponding author and published online for the benefit of the reader. The contents of supplementary data are the sole responsibility of the authors.

\section{REFERENCES}

1. Guan WJ, Ni ZY, Hu Y, Liang WH, Ou CQ, He JX, et al. Clinical characteristics of coronavirus disease 2019 in China. N Engl J Med. 2020;382:1708-20.

2. Watanabe M, Risi R, Tuccinardi D, Baquero C, Manfrini F, Gnessi $\mathrm{H}$. Obesity and SARS-CoV-2: a population to safeguard. Diabetes Metab Res Rev. 2020;e3325.

3. Yang J, Zheng Y, Gou X, Pu K, Chen Z, Guo Q, et al. Prevalence of comorbidities and its effects in patients infected with SARSCoV-2: a systematic review and meta-analysis. Int J Infect Dis. 2020;94:91-5.

4. Guan WJ, Liang WH, Zhao Y, Liang HR, Chen ZS, Li YM, et al. Comorbidity and its impact on 1590 patients with Covid-19 in China: a nationwide analysis. Eur Respir J. 2020;55:2000547.

5. Huang C, Wang Y, Li X, Ren L, Zhao J, Hu Y, et al. Clinical features of patients infected with 2019 novel coronavirus in Wuhan, China. Lancet. 2020;395:497-506.

6. Chen N, Zhou M, Dong X, Qu J, Gong F, Han Y, et al. Epidemiological and clinical characteristics of 99 cases of 2019 novel coronavirus pneumonia in Wuhan, China: a descriptive study. Lancet. 2020;395:507-13

7. Wang T, Du Z, Zhu F, Cao Z, An Y, Gao Y, et al. Comorbidities and multi-organ injuries in the treatment of COVID-19. Lancet. 2020;395:e52.

8. Datos Abiertos. Dirección General de Epidemiología. Secretaría de Salud. Available from: https://www.gob.mx/salud/documentos/datos-abiertos-bases-historicas-direccion-general-deepidemiologia. [Last accessed on $2020 \mathrm{Apr}$ 26].

9. Liu K, Fang YY, Deng Y, Liu W, Wang MF, Ma JP, et al. Clinical characteristics of novel coronavirus cases in tertiary hospitals in Hubei Province. Chin Med J (Engl). 2020;133:1025-31.

10. Wang D, Hu B, Hu C, Zhu F, Liu X, Zhang J, et al. Clinical characteristics of 138 hospitalized patients with 2019 novel coronavirus-infected pneumonia in Wuhan, China. JAMA. 2020; 323:1061-69.

11. Zhang JJ, Dong X, Cao YY, Yuan YD, Yang YB, Yan YQ, et al. Clinical characteristics of 140 patients infected with SARSCoV-2 in Wuhan, China. Allergy. 2020 [Epub ahead of print].

12. Gandhi RT, Lynch JB, Del Rio C. Mild or moderate Covid-19. N Engl J Med. 2020 [Epub ahead of print].

13. Onder G, Rezza G, Brusaferro S. Case-fatality rate and characteristics of patients dying in relation to COVID-19 in Italy. JAMA. 2020; [Epub ahead of print]

14. Blüher M. Obesity: global epidemiology and pathogenesis. Nat Rev Endocrinol. 2019;15:288-98.

15. Instituto Nacional de Salud Pública. Encuesta Nacional de Salud y Nutrición (ENSANUT). Mexico: Instituto Nacional de Salud Pública; 2018.

16. Zhou F, Yu T, Du R, Fan G, Liu Y, Liu Z, et al. Clinical course and risk factors for mortality of adult inpatients with COVID-19 in Wuhan, China: a retrospective cohort study. Lancet. 2020 395:1054-62.

17. Wu Z, McGoogan JM. Characteristics of and important lessons from the coronavirus disease 2019 (COVID-19) outbreak in China: summary of a report of 72,314 cases from the Chinese center for disease control and prevention. JAMA. 2020 [Epub ahead of print].

18. Ruan Q, Yang K, Wang W, Jiang L, Song J. Clinical predictors of mortality due to COVID-19 based on an analysis of data of 150 patients from Wuhan, China. Intensive Care Med. 2020; 46:846-8. 
19. Liu M, He P, Liu HG, Wang XJ, Li FJ, Chen S, et al Clinical characteristics of 30 medical workers infected with new coronavirus pneumonia. Zhonghua Jie He He Hu Xi Za Zhi. 2020; 43:209-14.

20. Peng YD, Meng K, Guan HQ, Leng L, Zhu RR, Wang BY, et al. Clinical characteristics and outcomes of 112 cardiovascular disease patients infected by 2019-nCoV. Zhonghua Xin Xue Guan Bing Za Zhi. 2020;48:E004.

21. Simonnet A, Chetboun M, Poissy J, Raverdy V, Noulette J, Duhamel A, et al. High prevalence of obesity in severe acute respiratory syndrome coronavirus-2 (SARS-CoV-2) requiring invasive mechanical ventilation. Obesity (Silver Spring). 2020 [Epub ahead of print].

22. Lighter J, Phillips M, Hochman S, Sterling S, Johnson D, Francois $F$, et al. Obesity in patients younger than 60 years is a risk factor for Covid-19 hospital admission. Clin Infect Dis. 2020;ciaa415

23. Zhou $P$, Yang $X$, Wang $X$, Hu B, Zhang L, Zhang $W$, et al. A pneumonia outbreak associated with a new coronavirus of probable bat origin. Nature. 2020;579:270-3.

24. Bourgeois C, Gorwood ], Barrail-Tran A, Lagathu C Capeau ], Desjardins D, et al. Specific biological features of adipose tissue, and their impact on HIV persistence. Front Microbiol. 2019; 10:2837.
25. Richardson S, Hirsch JS, Narasimhan M, Crawford JM, McGinn T, Davidson KW, et al. Presenting characteristics, comorbidities, and outcomes among 5700 patients hospitalized with COVID-19 in the New York city area. JAMA. 2020;323: 2052-59

26. Grasselli G, Zangrillo A, Zanella A, Antonelli M, Cabrini L, Castelli A, et al. Baseline characteristics and outcomes of 1591 patients infected with SARS-CoV-2 admitted to ICUs of the Lombardy region, Italy. JAMA. 2020;323:1574-81.

27. Epidemiology Working Group for NCIP Epidemic Response, Chinese Center for Disease Control and Prevention. The epidemiological characteristics of an outbreak of 2019 novel coronavirus diseases (COVID-19) in China. Zhonghua Liu Xing Bing Xue Za Zhi. 2020;41:145-51.

28. Wu C, Chen X, Cai Y, Xia J, Zhou X, Xu S, et al. Risk factors associated with acute respiratory distress syndrome and death in patients with coronavirus disease 2019 pneumonia in Wuhan China. JAMA Intern Med. 2020;e200994.

29. Dominguez-Cherit G, Lapinsky SE, Macias AE, Pinto R, EspinosaPerez L, de la Torre A, et al. Critically III patients with 2009 influenza A(H1N1) in Mexico. JAMA. 2009;302:1880-87.

30. Xie J, Toung Z, Guan X, Du B, Qiu H. Clinical characteristics of patients who died of coronavirus disease 2019 in China. JAMA Netw Open. 2020;3:e205619. 